DOE/ER/ $40344--5$

DE92 002279

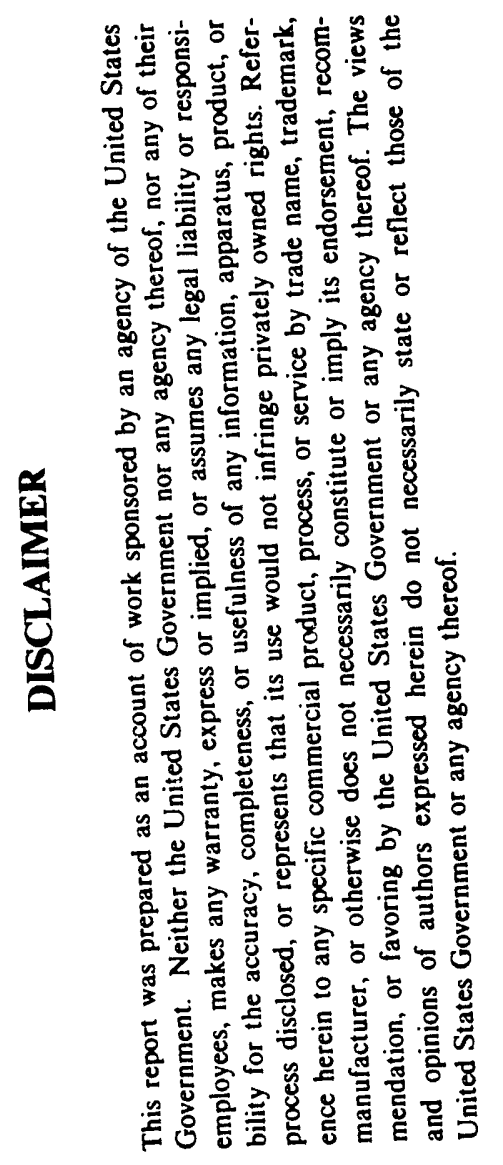

\title{
NUCLEAR PHYSICS STUDIES WITH MEDIUM ENERGY PROBES
}

Contract Period: 1989-92

Progress Report, Aug. 1990 - Aug. 1991

Budget, Dec. 1, 1991 - Nov. 30, 1992

Principal Investigator:

Kamal K. Seth

submitted to the

UNITED STATES DEPARTMENT OF ENERGY

NORTHWESTERN UNIVERSITY

Evanston, IL 60208

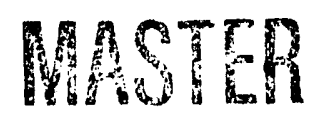

DISTRIBUTION OF THIS DOCUMENT IS UNLIMITED

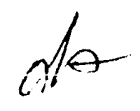




\section{CONTENTS}

1. INTRODUCTION 3

2. RESEARCH PROGRAMS 4

3. DATA ANALYSIS AND COMPUTERS 14

$\begin{array}{ll}\text { 4. PERSONNEL } & 15\end{array}$

5. PUBLICATIONS 16

6. INVITED PAPERS 18

$\begin{array}{lr}\text { 7. OTHER ACTIVITIES } & 19\end{array}$

8. BUDGET FOR 1991-92 、 20 -

9. REFERENCES 23 


\section{INTRODUCTION}

This progress report refers to the period September 1990 to September 1991 which includes the second year of the three year period, December 1, 1990 - November 30, 1992, of our existing research contract. The budget proposal for the year 1991-1992, as originally approved, is also presented.

During the last year the major activity of our research program was related to our Fermilab experiment E760 on antiproton production of charmonium and the study of $\bar{p} \bar{p}$ scattering between 3 and $9 \mathrm{GeV} / \mathrm{c}$. Our second run of data taking started in July ' 91 and is scheduled to continue through December '91. A report on the progress of this highly successful experiment, and the plans for its continuation are presented. Progress report of our research programs at LAMPF, Bates NIKHEF, and NSCL, and plans for the development and continuation of these programs, are also presented. 


\section{RESEARCH PROGRAMS}

\subsection{THE QUARK-QUARK INTERACTION - Fermilab Experiment E760}

It is generally accepted that QCD is the correct theory of strong interactions. However, until the time when lattice-gauge calculations are able to provide some insight into the problem, phenomenological models of QCD interactions must be used. In QCD, the quarkquark interaction is flavor-independent, and can be studied equally well between systems of any two quarks (or antiquarks). Unfortunately, relativistic corrections make the study of $q \bar{q}$ interactions between the light 'up', 'down' and 'strange' quarks very difficult. One must go to at least the charmed quarks to obtain meaningful answers. It is the objective of the Fermilab E760 experiment to make precision measurements of the energies, widths,

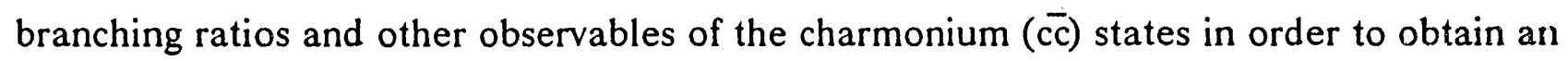
understanding of the $\mathrm{q} \overline{\mathrm{q}}$ interaction.

In the E760 experiment the charmonium states are populated directly by the annihilation of protons and antiprotons. This makes all the states with $\mathrm{J}^{\pi}=0 \pm, 1^{ \pm}, 2^{ \pm}$, and $3^{ \pm}$ accessible, in contrast to the $\mathrm{e}^{+} \mathrm{e}^{-}$experiments in which only $\mathrm{J}=1^{-}$states are directly produced. This makes E760 absolutely unique in its capabilities and it has been hailed as inaugurating "a new era in charmonium physics" (Jaffe[1]).

There is an extra dividend in the E760 experiment. Since charmonium states are produced in $\bar{p} \bar{p}$ collisions, it is possible to study forward angle $\bar{p} \bar{p}$ scattering in the $3 \mathrm{GeV} / \mathrm{c}$ $\rightarrow 9 \mathrm{GeV} / \mathrm{c}$ region at the same time as the charmonium measurements. Such measurements are extremely important if the nucleon-nucleon and the nucleon-antinucleon interactions are to be simultaneously understood at a fundamental (quark-gluon) level. The existing data in this energy region are extremely sparse and of very low quality.

The E760 experiment is located in the Fermilab antiproton accumulator in the middle of a straight section as shown in Fig. 1. The gas-jet target crossing the stochastically cooled beam and the surrounding detector system are located in a special pit-area constructed for the experiment.

As illustrated schematically in Fig. 2, the main detector consists of an annular detector system which covers a nearly $2 \pi$ solid angle around the beam/gas-jet intersection point. In 
brief, the various components of the system (and the main collaboration group responsible for each) are as follows:

Two Scintillation Hodoscopes $\mathrm{H} 1$ and $\mathrm{H} 2$ provide fast triggers in conjunction with the gas threshold Cerenkov counter.

The Threshold Cerenkov Counter is divided azimuthally into 8 identical segments. Each segment has two separate chambers with two different gases, $\mathrm{CO}_{2}$ in the forward chamber and Freon 13 in the back chamber.

The Central Calorimeter consists of 64 identical wedges, each consisting of 20 lead-glass blocks (16.4 to 12.5 radiation lengths), forming a cylindrical barrel which covers $10^{\circ}$ to $70^{\circ}$ in $\theta$, and $360^{\circ}$ in $\phi$. The rather fine grained segmentation $\left(1^{\circ}\right.$ to $5^{\circ}$ in $\theta, \sim 5^{\circ}$ in $\left.\phi\right)$ of this detector and the pointed geometry is meant to provide accurate determination of the centroids of the electromagnetic showers. This ability, coupled with the ability to detect $\gamma$ rays down to $\sim 30 \mathrm{MeV}$ energy, enables a high level of rejection of the background $\gamma$ 's from $\pi^{\circ}$ decays.

The Forward Calorimeter covers the angular region, $\theta=2^{\circ}-10^{\circ}$, and consists of a $12 \mathrm{x}$ 12 array of scintillation-glass and lead sandwich counters, each $10 \mathrm{~cm} \times 10 \mathrm{~cm} \times 48 \mathrm{~cm}$ deep. The Charged-Particle Tracking Counters consist of two rings of straw drift-tube detectors near the beam-pipe, the outer tracking chamber with two layers of Iarocci-tube detectors, and the so-called inner tracking chamber which consists of a radial projection chamber surrounded by a multiwire proportional counter. There is also an annular forward tracking charnber, consisting of three layers of multiwire proportional counters which provide charged particle tracking in the $\theta=10^{\circ}$ to $19^{\circ}$ region.

The Luminosity Monitor consists of a telescope assembly in which protons recoiling at $\theta=82^{\circ}$ to $90^{\circ}$ are detected in eight solid-state detectors. Of these eight detectors, two fixed detectors constitute the relative luminosity monitor. The other six detectors $(500 \mu, 3000 \mu)$, which are mounted on a movable carriage, are used for the small - $t$ elastic scattering measurements.

All items of the detector except the inner tracking chamber were operative in our June 1990-August 1990 run. The inner tracking chamber was successfully tested and installed early this year and the entire detector system has been satisfactorily operating during the 


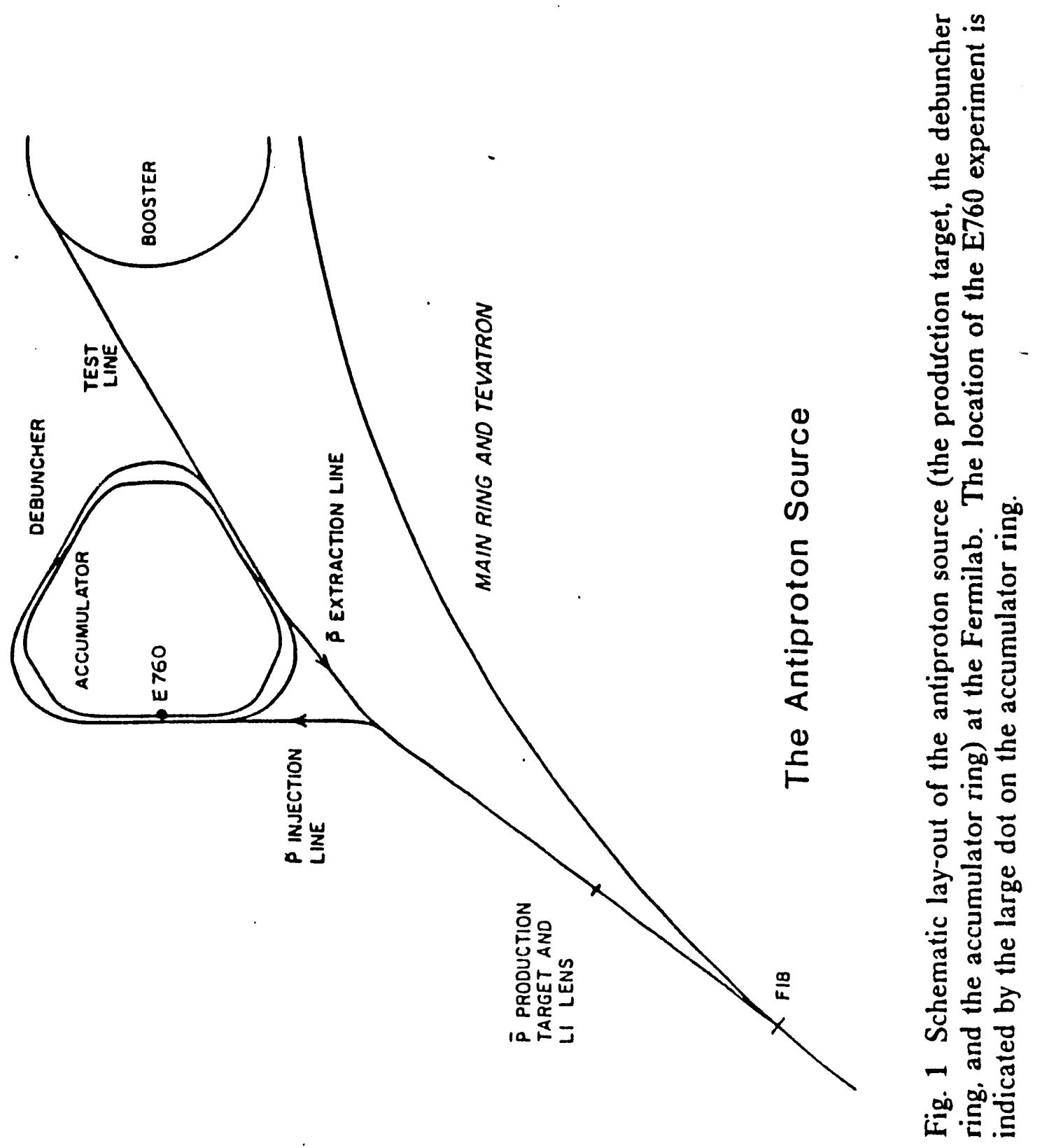




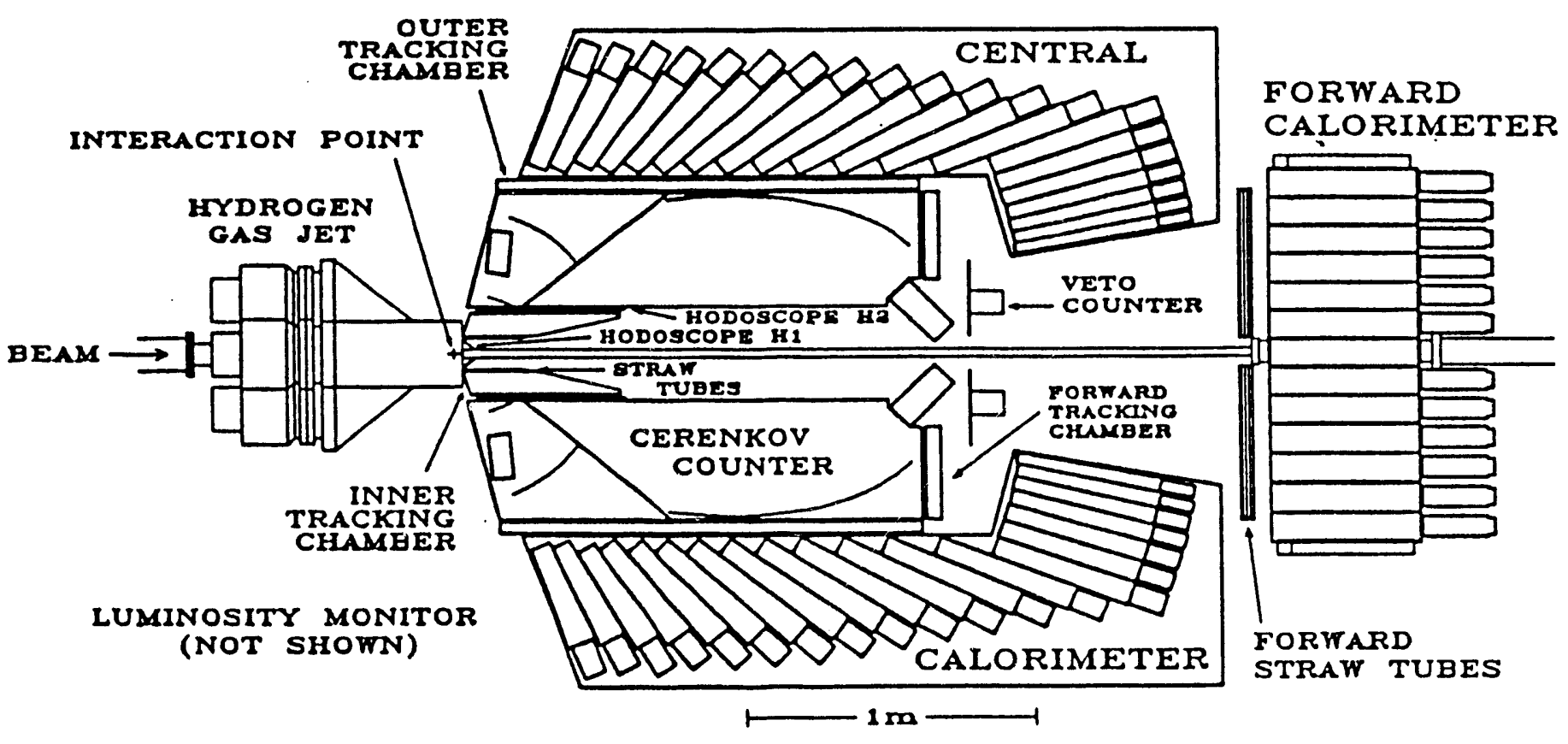

Fig. 2 Schematic representation of the E760 detector system. 
present run which started in July '91 and is expected to continue through December ' 91 .

Our group had the responsibility for the assembly and testing of the lead-glass central calorimeter. This responsibility was shared with colleagues from University of California (Irvine) and Fermilab. A paper describing the calorimeter has been published in "Nuclear Instruments and Methods"[2].

The $\bar{p} \bar{p}$ forward angle scattering measurements are the special interest and province of our group, and we were $100 \%$-responsible for the design, construction, testing and operation of the luminosity monitor. As described before, the luminosity monitor consists of an array of solid state detectors which 'look' at recoil protons at angles between $82^{\circ}$ and $90^{\circ}$. The detectors (each $1 \mathrm{~cm} \times 5 \mathrm{~cm} \times 500 \mu-3000 \mu$ ) are located in a pan at $\sim 1.5$ meters from the interaction point. The system comprises of two fixed detectors at $3.5^{\circ}$ and $4.5^{\circ}$ and six detectors located at $\sim 1^{\circ}$ intervals on a movable carriage (see Fig. 3 ). The pan is open to the vacuum of the accelerator $\left(\sim 10^{-10}\right.$ torr). With the gas-jet on the ambient pressure at the detectors is usually $\sim 10^{-8}$ torr. A remotely insertable ${ }^{244} \mathrm{Cm}$ sources is mounted at 1 meter from the detectors. It is used to monitor the performance of the detectors at regular intervals.

During the last year's operation some deterioration of the solid state detectors was observed in the high vacuum and radiation conditions prevailing in the luminosity monitor. Three detectors were affected. These were 'repaired' by the manufacturer, but their performance was found to be not satisfactory. We are currently operating with only five detectors. One of the problems we have encountered is that there are no U.S. detector makers who are willing to fabricate the detectors we need, and it is difficult to obtain replacements in time.

\subsection{PROTON-ANTIPROTON FORWARD SCATTERING}

During the last year and this year data have been taken at a number of charmonium resonances. This has enabled us to obtain $\mathrm{p} p$ forward angle elastic scattering data at the following momenta: 
Beam Momentum

Preliminary Analysis

$\begin{array}{lc}4.07 \mathrm{GeV} / \mathrm{c}(\mathrm{J} / \psi) & \text { Yes } \\ 5.55 \mathrm{GeV} / \mathrm{c}\left(\chi_{1}\right) & \\ 5.61 \mathrm{GeV} / \mathrm{C}\left({ }^{1} \mathrm{P}_{1}\right) & \text { Yes } \\ 5.72 \mathrm{GeV} / \mathrm{c}\left(\chi_{2}\right) & \text { Yes } \\ 5.87 \mathrm{GeV} / \mathrm{c}\left(\eta_{\mathrm{c}}{ }^{\prime}\right) & \\ 6.23 \mathrm{GeV} / \mathrm{c}\left(\psi^{\prime}\right) & \text { Yes }\end{array}$

$8.9 \mathrm{GeV} / \mathrm{c}$ (specifically for us)

This constitutes an enormous amount of data and will require a considerable amount of time for analysis. Such analysis is in progress and it is expected to continue throughout the next year. Preliminary result are already available at some of the energies, as indicated above. Two examples are shown in Fig. 3. It should be noted that the errors in our determinations of the parameters are factors 3-5 lower than the best results available in the literature. However, great care has to be exercised in the evaluation of systematic errors.

One of the rather exciting new results from our data is the value of $\rho$ parameter at 6.23 $\mathrm{GeV} / \mathrm{c}$. Our preliminary result is that $\rho=-0.030 \pm 0.014$. This is to be compared with the best earlier result at $6.0 \mathrm{GeV} / \mathrm{c}$ due to Jenni et al.[3], $\rho=0.040 \pm 0.060$. If the negative value of $\rho$ is confirmed by our 1991 data at $\psi^{\prime}$, it will constitute a very important point in the dispersion relations analysis of the global data. Such analyses almost 'require' a negative excursion by $\rho$ in order to accommodate the rather large positive value of $\rho$ measured at LEAR for energies below $0.8 \mathrm{GeV} / \mathrm{c}$ (see Fig. 4). The tendency for a possible negative excursion by $\rho$ is also indicated by the two old data points at 8.0 and $10.0 \mathrm{GeV} / \mathrm{c}$. However, these data have very large errors. In order to shed more light on this question we are presently taking data at our highest energy, i.e., $8.9 \mathrm{GeV} / \mathrm{c}$. 

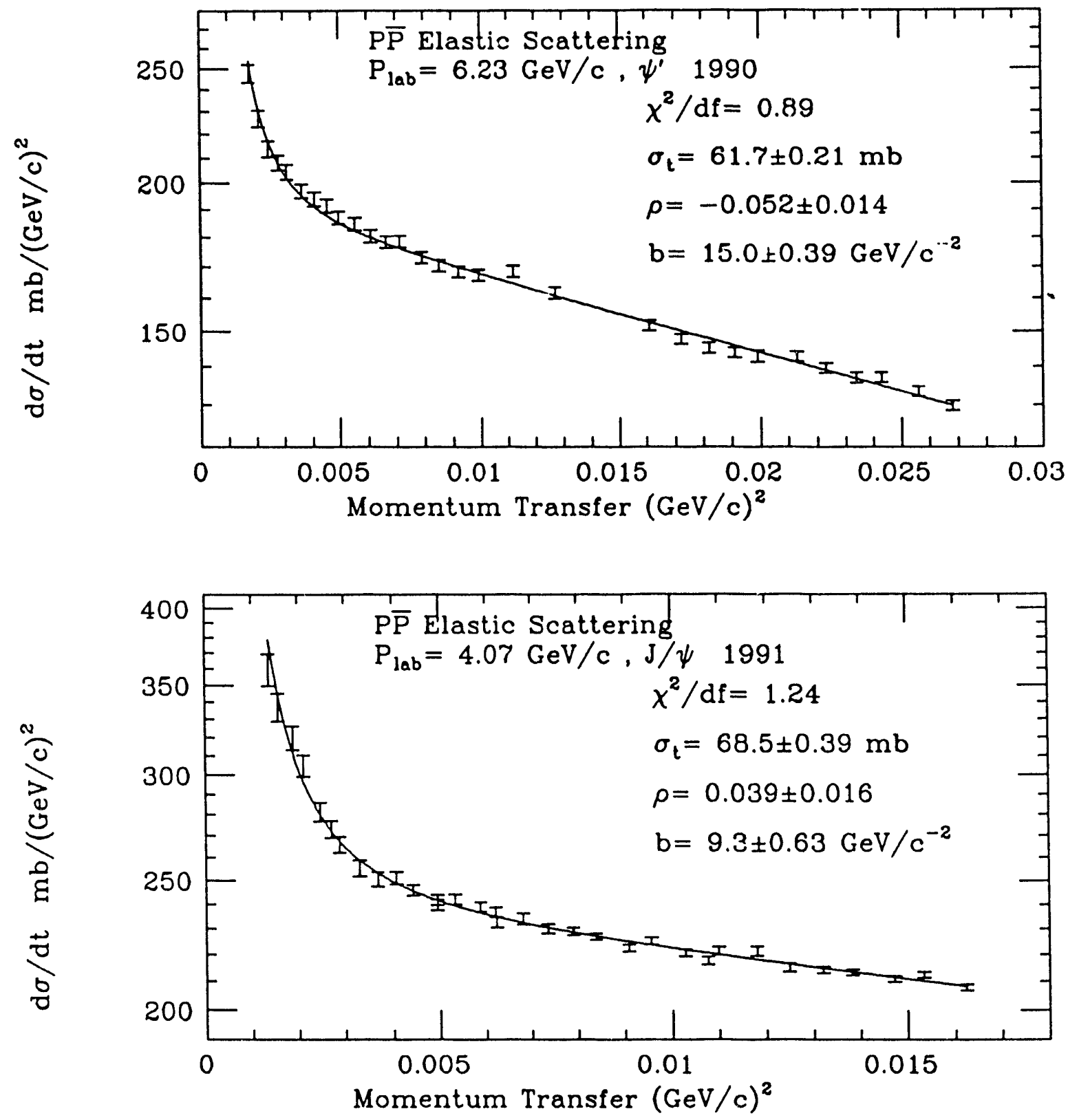

Fig. 3 Preliminary results for pp small-t elastic scattering at $4.07 \mathrm{GeV} / \mathrm{c}$ (top), and $6.23 \mathrm{GeV} / \mathrm{c}$ (bottom). 

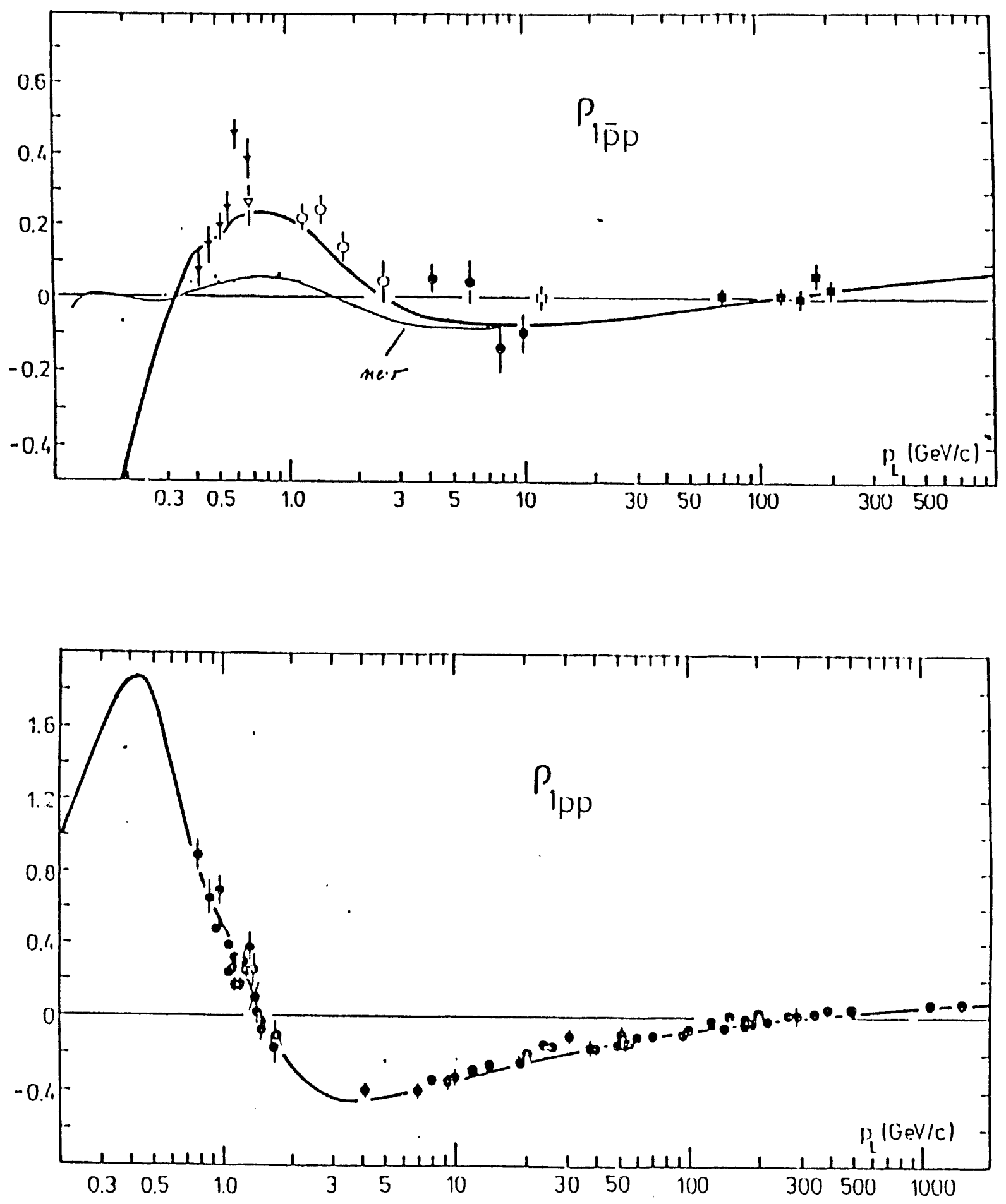

Fig. 4 The ratio $\rho$ of real to imaginary parts of the forward $p \bar{p}$ (top) and $\mathrm{pp}$ (bottom) scattering amplitudes. The solid lines are dispersion relations fits. Our preliminary results are indicated by open triangles. The errors are smaller than the size of the triangles. 


\subsection{CHARMC,NIUM SPECTROSCOPY}

As mentioned in the last progress report, the E760 detector is now fully operational. During the last year's running data was taken at the $J / \psi, \psi^{\prime}, \chi_{1}$ and $\chi_{2}$ resonarices, for the channel

$$
\mathrm{p} \dot{\mathrm{p}} \rightarrow(\mathrm{c} \ddot{c})_{\mathrm{R}} \rightarrow \mathrm{e}^{+} \mathrm{e}^{-}+(\gamma)
$$

For $\chi_{1}, \chi_{2}$ accurate measurements of the resonance parameters were made. The results, displayed below, represent immense improvement over the results of $\mathrm{e}^{-\mathrm{e}^{+}}$experiments, and even over the results of the E704 pp experiment done at CERN.[4]

\begin{tabular}{|c|c|c|c|}
\hline Resonance & $\mathrm{e}^{+} \mathrm{e}^{-}$expts. & p户́ expt. (R704) & $\mathrm{p} \overline{\mathrm{p}}$ results (ours) \\
\hline \multicolumn{4}{|c|}{ Total Widths } \\
\hline$\chi_{1}\left(1^{++}\right)$ & $<3.8 \mathrm{MeV}$ & $<1.3 \mathrm{MeV}$ & $0.88 \pm 0.11 \pm 0.08$ \\
\hline$x_{2}\left(2^{++}\right)$ & $2.8 \pm 2.1 \mathrm{MeV}$ & $2.6 \pm 1.1 \mathrm{MeV}$ & $1.29 \pm 0.09 \pm 0.13$ \\
\hline \multicolumn{4}{|c|}{$\underline{B R(R \rightarrow p \bar{p}) \text { in units of } 10^{-4}}$} \\
\hline$x_{1}\left(1^{++}\right)$ & $<12.0$ & $>0.54$ & $0.78 \pm 0.15$ \\
\hline$\chi_{2}\left(1^{++}\right)$ & 9.5 & $0.90 \pm 0.38 \pm 0.19$ & $0.92 \pm 0.15$ \\
\hline
\end{tabular}

These results have been submitted for publication in the Physical Review Letters[5], and also as a detailed communication in Nuclear Physics B[4].

In the 1990 data runs the $J / \psi$ and $\psi^{\prime}$ resonances were also scanned. Our energy resolution in $\sqrt{\mathrm{s}}$ is $\sim 300 \mathrm{keV}$, and this is at least an order of magnitude better than the best of $\mathrm{e}^{+} \mathrm{e}^{-}$experiments. However, even this resolution width is larger than the natural widths of the $\Psi(\Gamma \approx 67 \mathrm{keV})$ and $\psi^{\prime}(\Gamma \approx 240 \mathrm{keV})$ resonances. Therefore, great care has to be exercised in controlling beam characteristics and in data analysis. For these reasons it was decided to repeat the $\psi$ and $\psi^{\prime}$ scans during the present run-cycle using a new technique called the 'double scan' method. The data from these new measurements are being currently analyzed.

During the present run-cycle our main preoccupation has been to search for the missing 
${ }^{1} \mathrm{P}_{1}$ resonance and to verify the existence of the $\eta_{\mathfrak{s}}$. Both these investigations involve high luminosity searches around the expected energies. While the measurements are still in progress certain preliminary conclusions can be drawn.

Several possible decay channels for the ${ }^{1} \mathrm{P}_{1}$ state have been studied at five energy points in the vicinity of $3524 \mathrm{MeV}$, the center of gravity of the ${ }^{3} \mathrm{P}$ states. No evidence has so far been found the existence of the ${ }^{1} \mathrm{P}_{1}$ resonance. This is a great mystery.

Data have been taken at $\sqrt{\mathrm{s}}=3.590 \mathrm{GeV}$ where the Crystal-Ball group[7] has claimed to find the $\eta_{c}^{\prime}$ resonance. This energy is at least $30 \mathrm{MeV}$ lower than the theoretical expectations. We have been doing scans around this energy but have so far found no evidence for any enhancement above backgroun

There are many other decay channels, e.g., $(c)_{R} \rightarrow 2 \gamma, 3 \gamma, \cdots 6 \gamma$ which are also being studied. Most of the analysis is currently in a very preliminary stage. Nevertheless, it is clear that there is a great wealth of new information in our data. We expect to be able to report on several interesting results in light meson spectroscopy, proton time-like form factor, and QCD-scaling laws in the near future.

At the end of the present run-cycle, expected to be $\sim$ December 15,1991 , Fermilab will go into a year-long cycle of colliding beam runs. During this period E760 cannot take data. Besides doing the analysis of the immense amcunt of data which have been acquired during this run-cycle, this period will also be used to plan for the upgrade of the E760 detector and for the physics to be pursued during the next run-cycle.

It is clear that a number of the original objectives of the E760 experiment will remain unfulfilled at the end of the present run-cycle. These will, of course, constitute the first priority for the next run. However, it is expected that there will be beam-time to extend the physics scope of E-760 beyond charmonium spectroscopy. Many ideas for the new physics are being currently examined. Among these is our proposal to study charmonium in nuclei.

$\mathrm{J} / \Psi$ Production in Nuclei: Knowledge of $\mathrm{J} / \Psi$-nucleon cross section is of fundamental importance. It is also needed to evaluate purported signals of quark-gluon plasma formation in heavy-ion collisions. The present knowledge of $\mathrm{J} / \Psi$-nucleon cross sections is essentially non-existent. The commonly used value, $2 \pm 1 \mathrm{mb}$, comes from photon-nucleus experiments 
at several hundred $\mathrm{GeV} / \mathrm{c}$. It has been pointed out by Brodsky and Müller,[8] and many other authors, that because of large color-transparency effects at these high momenta, this value has nothing to do with the real $J / \psi$-nucleon cross section. In order to measure it, it is necessary that the process of $J / \psi$ formation be a low momentum transfer process so that color-transparency effect is negligibly small. $\mathrm{J} / \Psi$ production in $\mathrm{p} \overline{\mathrm{p}}$ annihilation inside a nucleus is ideally suited for the purpose. We therefore propose to study $J / \psi$ resonant production in nuclei using the E760 detector.

Several improvements and changes have to be made to the present E760 experiment in order to be able to study $\mathrm{J} / \Psi$ production in nucle:. A nuclear-gas jet-target has to be designed. Beam life-time studies with such a jet-target have to be studied. Luminosity monitoring technique has to be refined. Finally, strategies have to be developed to make these measurements without making unreasonable demands on beam-time.

We are developing this proposal in collaboration with our colleagues at the Universities of Torino, Genoa and Ferrara. Work on the nuclear-gas jet-target development is being done in collaboration with University of Genoa. In order to work on this program in the most efficacious manner, the Principal Investigator (K.K. Seth) plans to spend most of the calendar year, January 1991 to January 1992 in Italy. Northwestern University has approved a leave of absence for the purpose, and the Istituto Nazionale di Fisica Nucleare (INFN), Italy has extended him an appointment as a Visiting Professor.

\subsection{EXOTIC BARYRONIC SYSTEMS}

In this proposed experiment, which is ideally suited for existing heavy-ion beams at the SPS at CERN, we wish to study the stability of single- and double-lambda hypernuclei, sigma hypernuclei, cascade hypernuclei and even omega minus-neutron systems. The basic idea of the experiment is to look for these nuclei containing strangeness in the products of collision of a (relatively light) heavy-ion beam of $>200 \mathrm{GeV} /$ nuclear with a heavy nucleus target. It is expected that the forward going fragmentation products will include strangeness containing light 'nuclei'. Systems having unusual charge/mass ratios can be selected by their passage through a high field magnet (the survival of the strangeness containing 'nuclei' is 
insured by the large $\gamma$-factor). The emerging 'nuclei' are to be then identified by their characteristic decays.

We are at present exploring the possibility of a joint project for this experiment between GSI, Heidelberg and Northwestern. It is expected that the formation of a collaboration and preparation of a formal proposal for this experiment will be greatly facilitated by the presence of the Principal Investigator in Europe during the next year.

\subsection{NUCLEAR STRUCTURE EXPERIMENTS}

\subsection{PION DOUBLE CHARGE EXCHANGE}

Our program of pion double charge exchange experiments at LAMPF was continued during the year 1990-91, albeit with rather limited success. Our experiment (LAMPF 1198), "Correlation Effects in Analog DCX on Nickel Isotopes" was scheduled for one month of beam-time during this summer. After numerous postponements it got three weeks of beamtime from June 26-July 20. The LAMPF accelerator performance during the period was extremely poor. The beam intensity was approximately half of the usual, and the beam availability was also only $\sim 50 \%$. The net result was about $1 / 4$ the pion luminosity expected. Data was therefore taken only on one nickel isotope, ${ }^{58} \mathrm{Ni}$, and not on the other three, ${ }^{60} \mathrm{Ni}$, ${ }^{62} \mathrm{Ni}$, and ${ }^{64} \mathrm{Ni}$, and we were informed that we will be rescheduled next year.

The inability to complete the experiment during the last year has produced another problem. We need to retain the isotopic targets in their present metallic form for use next year. However, with the new rules and pricing structure for loans imposed by the ORNL Stable Isotopes Pool, we cannot afford to do so. We have requested LAMPF to assume the loans. If they are not able to do so, this experiment, among others, will have to be scrapped. This will be a serious blow, not only to our program but to pion physics. 


\subsection{EXOTIC NUCLEI}

Our studies of light exotic nuclei by means of pion DCX reactions continue to be very fruitful. Following are the highlights.

(a) The results of our experiment on the reaction ${ }^{6} \mathrm{Li}\left(\left(\pi^{-}, \pi^{+}\right)^{6} \mathrm{H}\right.$ were published in Physics Letters[9]. We showed that there is no evidence for ${ }^{6} \mathrm{H}$ at the level of $\mathrm{d} \sigma / \mathrm{d} \Omega<2 \mathrm{nb} / \mathrm{sr}$. This negative result does not, of course, establish that the Soviet claims[10] of having discovered ${ }^{6} \mathrm{H}$ in heavy-ion reactions are wrong. We have therefore proposed to repeat the Soviet experiment on the reaction ${ }^{7} \mathrm{Li}+{ }^{7} \mathrm{Li} \rightarrow{ }^{8} \mathrm{~B}+\mathrm{X}$ under much better conditions at the NSCL at the Michigan State University. The experiment has been approved and currently awaits scheduling[11].

(b) Recently, a powerful new technique for creating and studying the exotic light nuclei was invented by Garnov and his colleagues at Leningrad[12]. The method essentially consists of absorbing stopped $\pi^{-}$in light nuclei and detecting coincident fragments, p,d,t, ${ }^{3} \mathrm{He}$ and ${ }^{4} \mathrm{He}$ in two solid-state detector telescopes. Thus, for example, the detection of two coincident charged particles in the reactions ${ }^{9} \mathrm{Be}\left(\pi^{-}, \mathrm{dt}\right)^{4} \mathrm{H}, \quad{ }^{9} \mathrm{Be}\left(\pi^{-}, \mathrm{pt}\right)^{5} \mathrm{H}, \quad{ }^{9} \mathrm{Be}\left(\pi^{-}, \mathrm{pd}\right)^{6} \mathrm{H}$ leads to the missing mass spectra of ${ }^{4} \mathrm{H},{ }^{5} \mathrm{H}$ and ${ }^{6} \mathrm{H}$. Because of the excellent energy resolution achieved in this type of experiment, it is very well suited for measuring the masses and excited state spectra of exotic nuclei. Also, as illustrated above several nuclei can be studied at the same time.

The experiments at Leningrad are limited by the extremely low pion fluxes available at the Synchrocyclotron. Garnov et al. have approached us to form a collaboration and do the much improved experiments which are possible at LAMPF with a factor 100 larger pion flux. They have proposed to do so under the auspices of the US-USSR Collaborative Scientific Research Program. We are currently developing a joint proposal for this project.

(c) We have studied the continuum spectra associated with the $\left(\pi^{-}, \pi^{+}\right)$and $\left(\pi^{-}, \mathrm{p}\right)$ reactions leading to extremely neutron-rich weakly-unbound systems. We find that 
these spectra can unly be explained if it is assumed that a dineutron is present among the products of the breakup. On the basis of these observation we conclude that 'dineutrons' are present in the tail-(or halo-) part of the density distributions for these extremely neutron-rich systems. These results were recently published in the Physical Review Letters[13].

\subsection{HIGH RESOLUTION ELECTRON SCATTERING}

The final analysis of our NIKHEF data on high resolution inelastic scattering of electrons from ${ }^{26} \mathrm{Mg}$ has now been completed for 75 states up to an excitation of $9 \mathrm{MeV}$. Form-factors and model independent transition densities have been obtained for all the transitions. Two papers describing the detailed experiment results and their comparison with shell model calculations[14] are under preparation.

\subsection{DEEP INELASTIC ELECTRON SCATTERING}

In this experiment we have measured deep inelastic scattering of electrons from targets of ${ }^{40} \mathrm{Ca},{ }^{48} \mathrm{Ca}$ and ${ }^{238} \mathrm{U}$. Data were taken at several angles for incident electron energies between 400 and $850 \mathrm{MeV}$, in order to permit Rosenbluth separation of longitudinal and transverse spectral functions. Considerable progress has been made in the analysis of the data. Preliminary results indicate that we do not observe the strong quenching of the longitudinal response function as reported by Saclay.

In view of the dramatic discrepancy between the Saclay results and our present (as well as earlier published) results, we feel that it is necessary to do a carefully designed new series of improved experiments at Bates. Accordingly, we submitted a Letter of Intent to the Bates PAC (copy attached). Their repsonse was encouraging. As a result, we have been invited to discuss our plans with the Director of Bates. We intend to do so in the very near future. 


\section{DATA ANALYSIS AND COMPUTERS}

Our VAX-750 computer system and its disc-storage system was decommissioned early this year. Our entire computing load is now borne by a Vaxstation 3520 and a Vaxstation 3100. While we still maintain the capability of reading $6250 \mathrm{bpi}, 10$ " reel tapes (because data acquisition at LAMPF is still done on those tapes) we have acquired two Exabyte drives and use them for processing of all our Fermilab data. Similarly, we have acquired a $702 \mathrm{MB}$ and a $1.4 \mathrm{~GB}$ SCS1 disc to replace the old RA81 system. It is intended to add an additional 1.4 GB disc in the coming year. 


\section{PERSONNEL}

(September 1989 - September 1990)

Kamal K. Seth, Principal Investigator

Dimitri Dimitroyanisis, Research Associate

Miguel Sarimento, Research Associate

Steve Trokenhiem, Graduate Student

Camille Ginsburg, Graduate Student

Todd Pedlar, Summer Graduate Student

David Hahn, Summer Graduate Student 


\section{PUBLICATIONS}

(August 1990 - August 1991)

1. "Search for Superheavy Hydrogen-6", B. Parker, Kamal K. Seth, and R. Soundranayagan. Phys. Lett. B251 (1990) 483.

2. "Dineutrons in Extremely Neutron Rich Nuclei", Kamal K. Seth and B. Parker, Phys. Rev. Lett. 66 (1991) 2448.

3. "Nuclear Physics with Pions", Kamal K. Seth, Proc. VI International School of Intermediate Energy Physics (Venice), edited by S. Costa et al., (World Scientific, Singapore, 1990), pp. 43-125.

4. "Fun and Games with DCX." Kamal K. Seth, Proc. Second LAMPF Inte. Workshop on Pion Double Double Charge Exchange (Los Alamos), edited by W. Gibbs (World Scientific, Singapore 1990), pp. 473-501.

5. "Neutron-Rich Exotic Nuclei" in section on Nuclear Physics in McGraw-Hill Encyclop edia of Science and Technology, edited by D. Bromley (McGraw-Hill Publishers), in press.

6. "Do the (Neutron-) Rich (Nuclei) Have a Halo", Kamal K. Seth, Proc. VII International School of Intermediate Energy Physics (L'Áquila 1990), edited by S. Costa et al. (World Scientific, Singapore), pp. 1-29.

7. "Pions in Pursuit of the Exotic", Kamal K. Seth, Proc. International Workshop on Pions in Nuclei" (Peñiscola 1991), edited by E. Oset (World Scientific) pp. 1-35.

8. "Nuclear Physics with Charm", Proc. Lake Lousie Winter Institute, Lake Louise, (World Scientific, Singapore, 1991) pp. 1-20.

9. "The E760 Lead-Glass Central Calorimeter, L. Bartoszek et al., Nucl. Instr. Methods A301 (1991) 47.

10. "A Luminosity-Independent Measurement of $\mathrm{pp}$ Total Cross Section at $\sqrt{\mathrm{s}}=1.8$ TeV," N.A. Amos et al. (The E710-collaboration), Phys. Lett. B243 (1990) 158.

11. "Inclusive Neutrai D0 Production in $205 \mathrm{GeV} / \mathrm{c} \pi^{-}$-Be Interactions" M. Sarmiento et al., submitted to Phys. Rev. D. 
12. "Measurement of $\rho$, the Ratio of the Real to Imaginary Part of the pp Forward Elastic Amplitude, at $\sqrt{\mathrm{s}}=1.8 \mathrm{TeV}$ ", N.A. Amos et al., (The E710 collaboration), submitted to Phys. Lett. B.

13. "Precision Measurements of Charmonium States in the Fermilab Accumulator, T. Armstrong et al., submitted to Phys. Rev. Lett.

14. "Study of $\chi_{1}$ and $\chi_{2}$ Charmonium States formed in pp Annihilations", T. Armstrong et al., submitted to Nucl. Phys. B. 
6. INVITED PAPERS (Kamal K. Seth)

1. "A Review of the Experimental Status of Non-Strange Dibaryons", Rheinfels '90 Workshop on Hadron Spectroscopy, Rheinfels, Germany, 1990.

2. "High Resolution Charmonium Spectroscopy with Antiprotons", Annual Meeting Division of Nuclear Physics, American Physical Society, Champaign, IL. 1990.

3. "Antiproton-Nucleus Physics" Lake Louise Winter Institute, Lake Louise, Canada 1991.

4. "Testing QCD with Antiprotons" Hadron '91, 4th International Conference on Hadron Spectroscopy, College Park, Md., 1991.

5. "Antiproton Reactions and Charm within and without Nuclei" 13th European Conf. on Few-Body Problems in Physics, Elba, Italy, 1991.

6. "Antiproten-Nucleus Experiments" Workshop on Physics at SuperLear, Zurich, Switzerland, 1991. 


\section{OTHER ACTIVITIES (Kamal K. Seth)}

- Author: invited review article on "DIBARYONS" for Reviews of Modern Physics (AIP), in preparation.

- Author: invited review article on "PION PHYSICS" for Progress in Nuclear and Particle Physics (Pergamon Press), in preparation.

- Member International Advisory Committee

- Workshop on Nuclean-Antinculeon Interactions (ITEP), Moscow, USSR

- International Workshop on Pions in Nuclei, Penyscola, Spain

- 13th International Conf. on Few Body Problems in Physics, Adelaide, Australia

- Reviewer: research papers for Physical Review Letters, Physics Letters and Nuclear Physics

- Reviewer: research proposals for the Department of Energy and the National Science Foundation 


\section{BUDGET (1991-92)}

We reproduce below the budget for the contract period December 1, 1991 - November 30, 1992, as approved in our three year (1989-1992) renewal last year. As mentioned elsewhere in this report, the Principal Investigator plans to spend a major part of the next contract year in Italy (primarily at the University of Torino) for the exclusive purpose of doing research related to this program. For this purpose he has been granted leave by the Northwestern University. It is intended that he will make several long-term (1-2 months each) trips to Italy, with intervening short stays at Northwestern. Larger periods of stay at Los Alamos and Bates may be required as needed for scheduled experiments. It is proposed that for location in Torino, where an apartment will be maintained, only the lodging expenses (no subsistence) will be charged to the contract. Other travel expenses will be treated as usual. Because of the larger than anticipated expenses in foreign travel, and correspondingly smaller expenses in domestic travel, the approved travel funds have been partitioned between the two categories differently than before. No other changes have been made to the originally approved budget.

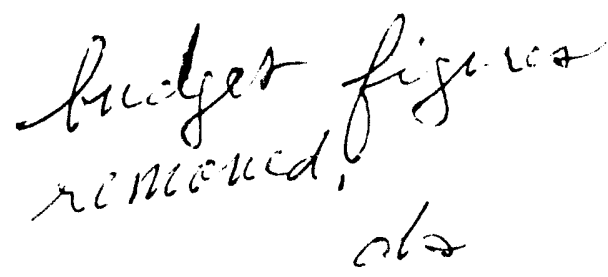




\section{REFERENCES}

1. R.L. Jaffe, Proc. 1st Workshop on Antimatter Physics at Low Energies, Fermilab, 1986, edited by B.E. Bonner and L.S. Pinsky, 1986.

2. L. Bartoszek et a!., Nucl. Instr. Methods A301 (1991) 47.

3. P. Jenni et al, Nucl. Phys. B129 (1977) 232.

4. C. Baglin et al., Nucl. Phys. B286 (1987) 592.

5. The E760 Collaboration (T. Armstrong et al.), Phys. Rev. Lett., submitted for publication.

6. The E760 Collaboration (T. Armstrong et al.,) Nucl. Phys. B, submitted for publication.

7. C. Edwards et al., Phys. Rev. Lett. 48 (1982) 70.

8. S.J. Brodsky and A.H. Muller, Phys. Lett. B206 (1988) 685.

9. B. Parker, Kamal K. Seth, and R. Soundranayagam, Phys. Lett. 251 (1990) 483.

10. D.V. Aleksandrov et al. Sov. J. Nucl. Phys. 39 (1984) 323; A.V. Belozyorev et al., Nucl Phys. A460 (1986) 352.

11. "Does ${ }^{6} \mathrm{He}$ Really Exist", a proposal submitted to the National Superconducting Cyclotron Laboratory, spokesman: Kamal K. Seth.

12. M.G. Gornov et al., Nucl. Instr. Methods 225 (1984) A42; also Yad. Fiz. 47 (1988) 959, 47 (1988) 1193; also priv. comm.

13. Kamal K. Seth and B. Parker, Phys. Rev. Lett. 66 (1991) 2448.

14. B.A. Brown, R. Radhi, and B.H. Wildenthal, Phys. Rep. 101 (1983) 313; also priv. comm. (1990).

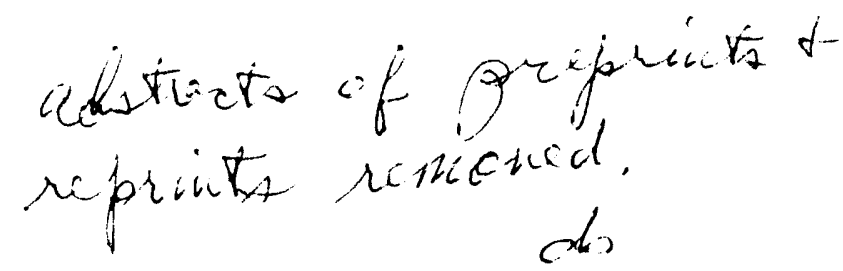



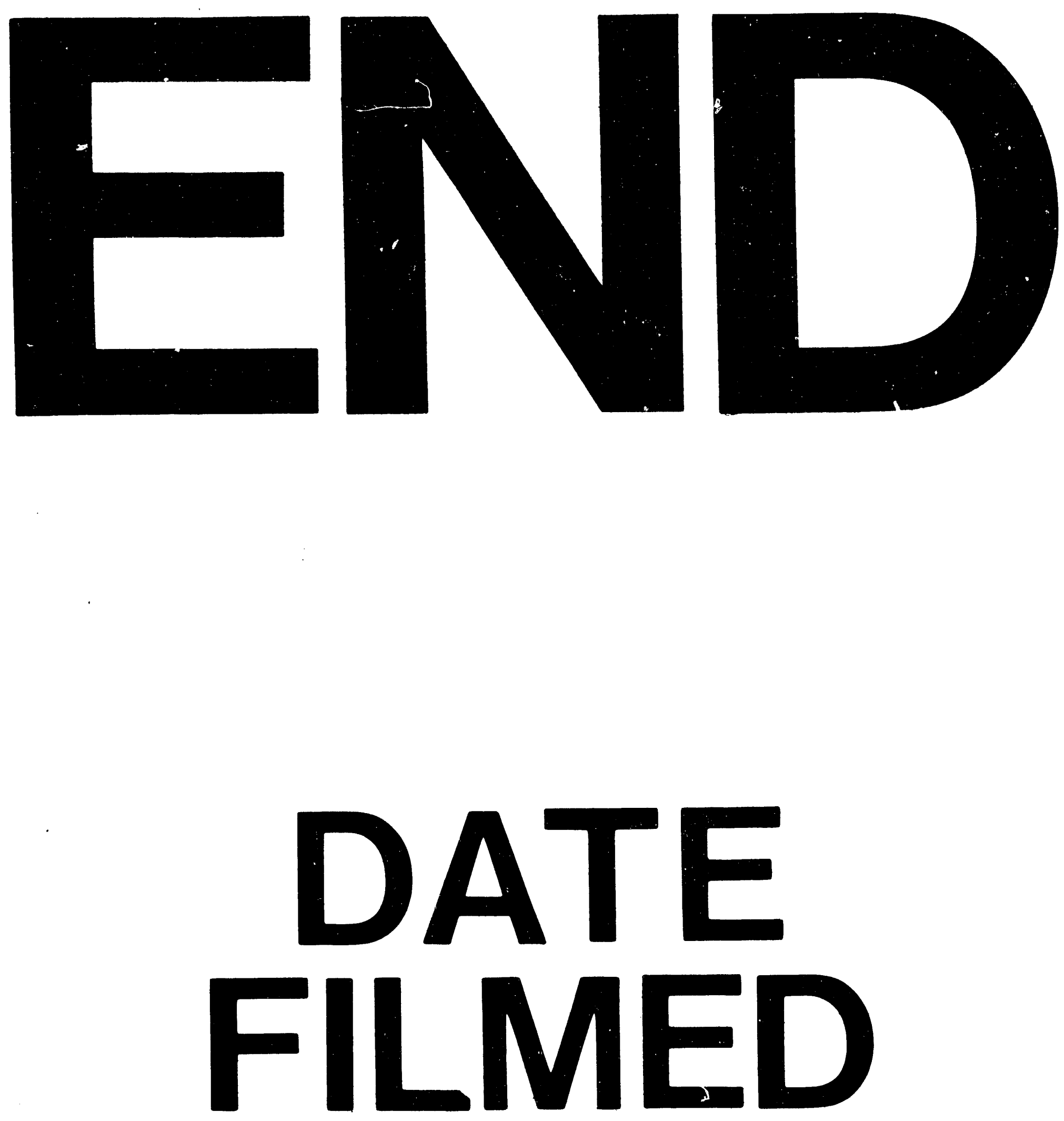

I

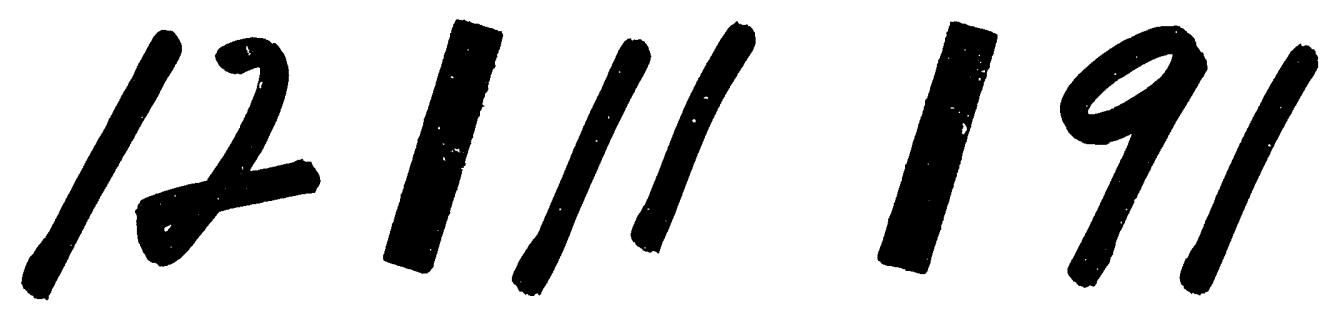

